\title{
Influence of blood contamination before or after surface treatment on adhesion of 4-META/MMA-TBB resin to root dentin
}

\author{
Hiroe TAKEFU, Shinji SHIMOJI, Tsutomu SUGAYA and Masamitsu KAWANAMI \\ Department of Periodontology and Endodontology, Division of Oral Health Science, Hokkaido University Graduate School of Dental Medicine, N13 \\ W7 Kita-ku, Sapporo, Hokkaido 060-8586, Japan \\ Corresponding author, Hiroe TAKEFU; E-mail: fukuhiro@den.hokudai.ac.jp
}

\begin{abstract}
The purpose of this study was to evaluate the influence of blood contamination before or after surface treatment on adhesion of 4-META/MMA-TBB resin. After bovine root dentin surfaces were contaminated with blood before or after dentin surface treatment with 10-3 solution, the contaminated surface was rinsed with water, air-dried, or re-treated with 10-3 solution. Dye leakage and microtensile bond strength (MTBS) of 4-META/MMA-TBB resin to dentin were measured after storage in water for $24 \mathrm{~h}$. When blood contamination occurred before surface treatment, there was no significant difference in the leakage value and MTBS as compared with that of the uncontaminated group. When blood contamination occurred after surface treatment, the leakage value increased and MTBS significantly decreased $(p<0.05)$ even if the blood was washed away. However, when the surface was re-treated with $10-3$ solution after rinsing with water, the leakage value and MTBS were restored to those of the uncontaminated group.
\end{abstract}

Keywords: 4-META/MMA-TBB resin, Blood contamination, Leakage, Microtensile bond strength, Surface treatment

\section{INTRODUCTION}

The diffusion and polymerization of adhesive monomers into the demineralized dentin layer and the formation of a hybrid layer are important to achieve durable adhesion between dentin and adhesive resin ${ }^{1-4)}$. However, in the clinical situation, several factors such as gingival fluid, saliva and blood contaminants reduce the adhesive property. Some studies have reported that blood contamination particularly impaired the adhesion between dentin and resin ${ }^{5-13)}$.

4-methacryloxyethyl trimellitate anhydride/methyl methacrylate-tri- $n$-butylborane (4-META/MMA-TBB) resin has been widely used for bonding crowns, restorations, orthodontic brackets and temporary splints for periodontal disease because of its remarkable long-term adhesive properties to enamel and dentin ${ }^{2,3,14}$. Furthermore, because of its high biocompatibility to dental pulp and periodontal tissue ${ }^{15-17)}$, it has been recently applied for direct pulp capping ${ }^{18)}$, retrograde root-end sealing following apicoectomy or intentional replantation ${ }^{19,20)}$, sealing of perforation sites and the bonding treatment of vertically fractured $\operatorname{roots}^{21,22)}$. These procedures often induce operative hemorrhage and blood contamination of the adherent dentine surface, which result in inferior bonding and increased risk of resin detachment. However, the influence of blood contamination on adhesion of 4-META/MMA-TBB resin to dentin has to be further clarified ${ }^{5,6)}$.

On the other hand, several studies have investigated the influence of blood contamination on adhesion of other restorative resins ${ }^{7 \cdot 13)}$.

Some studies on a conventional three-step adhesive system, which consisted of acid etchant, primer and adhesive, revealed that blood contamination at any step of the adhesive process reduced the bond strength ${ }^{5,7-9,12)}$.
The result suggested that residual blood contaminants might interfere with priming and resin impregnation into decalcified dentin ${ }^{7-9)}$.

Another study using the wet bonding system, in which the dentin surface was not dried after acid-etching and fewer collagen fibers were collapsed, indicated that the bond strength was less sensitive to contamination before etching ${ }^{12}$.

As for the self-etching adhesive system, it was reported that blood contamination before primer application did not strongly affect the bond strength after washing because the acidic primer itself could clean the blood and highly penetrate into the low-decalcified dentin ${ }^{12,13)}$.

These reports suggest that the influence of blood contamination on resin adhesion differs with dentine adhesive systems, the timing when the contamination occurred in the adhesive process (e.g., before or after the surface treatment) and so on.

In addition, it was shown that with self-adhesive resin cement, which contains acidic phosphate monomer (MDP), blood contamination of the adherent surface can influence the bond strength to dentin. When blood contamination occurred before the collagen fibers were exposed by either phosphoric acid etching or self-etching primer application, the bond strength was not affected. However, blood contamination of the dentin surface where collagen fibers had been exposed decreased the bond strength ${ }^{10)}$. These results revealed that the influence of blood contamination differed with the adherent surface conditions.

4-META/MMA-TBB resin is a self-cure adhesive resin cement based on MMA with a monomer 4-META and a catalyst TBB. The dentin surface is treated with an aqueous solution of $10 \%$ citric acid and $3 \%$ ferric chloride that removes the smear layer and, at the same 
time, stabilizes the exposed collagen ${ }^{23)}$. These may explain the difference in the influence of blood contamination on adhesion from other resins.

The purpose of this study was to evaluate the influence of blood contamination on the leakage and bond strength of 4-META/MMA-TBB resin to dentin.

\section{MATERIALS AND METHODS}

\section{Specimen preparation}

Twenty-four extracted bovine incisors were used in this study. 4-META/MMA-TBB resin (SB; Super Bond C\&B ${ }^{\circledR}$, clear type, Sun Medical, Shiga, Japan) was used as the adhesive resin cement (Table 1). Fresh capillary blood obtained from one of the authors was used without anticoagulants as the contaminant. This study was approved by the Medical Research Ethics Commission of Graduate School of Dental Medicine, Hokkaido University.

After superficial soft tissue and cementum were removed from the root of each tooth with a curette-type scaler, the crown was cut off at the cemento-enamel junction with a low-speed diamond saw (Isomet, Buehler, Lake Bluff, IL, USA). The root pulp was removed with a barbed broach. The exposed surface was then polished with 600-grit silicon carbide paper to standardize the superficial morphology. Six specimens were obtained from one root. Thirty-six specimens were used for SEM observation of the dentin surface, 60 specimens for the dye leakage test and SEM observation of the adhesive interface, and 48 specimens for the microtensile bond strength test.

Table 1 Materials used in this study

\begin{tabular}{llc}
\hline Products/Materials & \multicolumn{1}{c}{ Composition } & Manufacturer \\
\hline Super-Bond C\&B & & Sun Medical, Shiga, Japan \\
Monomer & $5 \%$ 4-META, MMA & \\
Catalyst & TBB \\
Polymer & PMMA \\
Green Activator & $10 \%$ citric acid, 3\%ferric chloride, water \\
Bovine teeth & & \\
Human blood & & \\
\hline
\end{tabular}

4-META: 4-methacryloxyethyl trimellitate anhydride; MMA: methyl methacrylate; TBB: tri- $n$-butylborane; PMMA: polymethyl methacrylate.

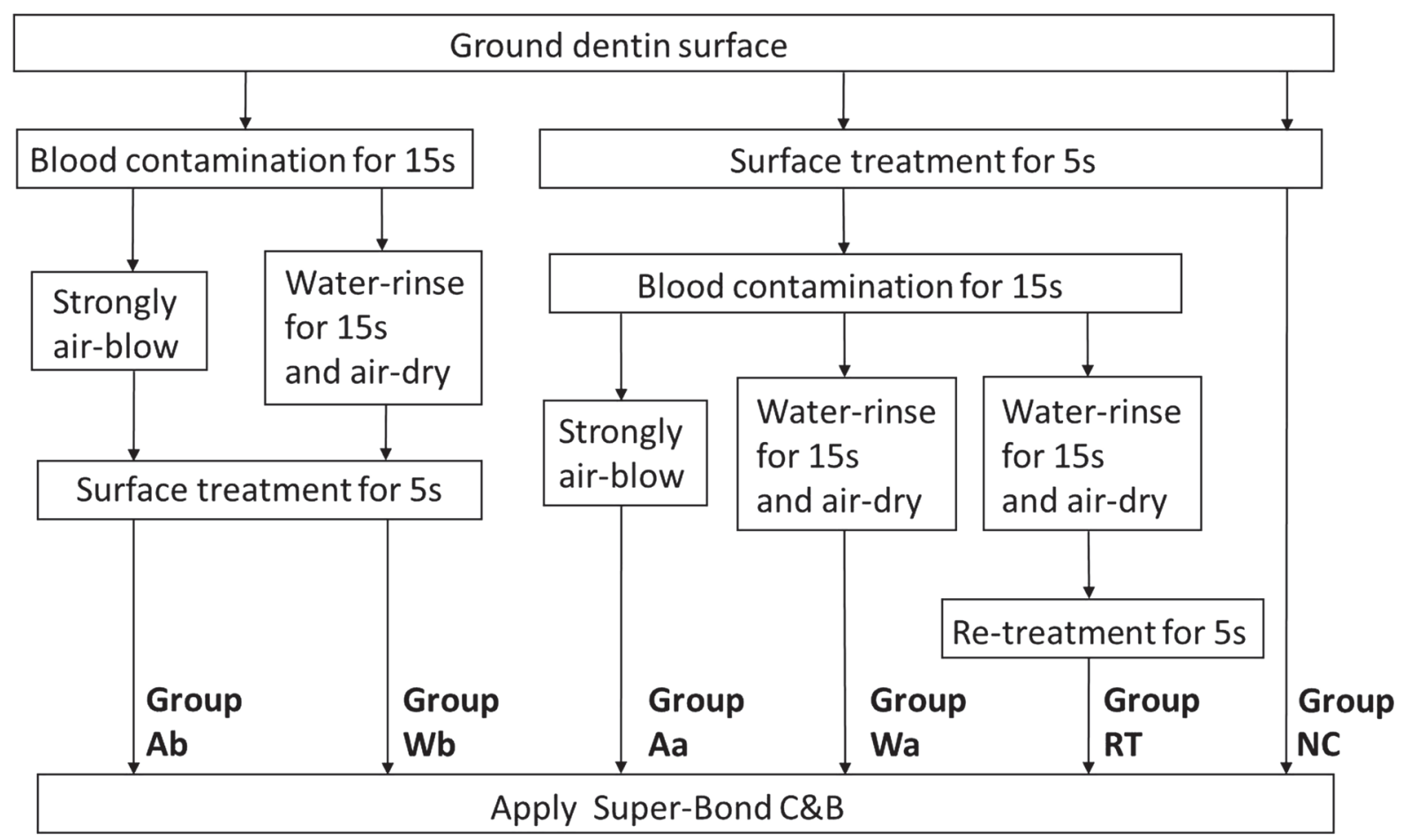

Fig. 1 Blood contamination and adhesive procedures. 
Blood contamination and adhesive procedures

Specimens were randomly divided into six groups, as described below (Fig. 1).

1. Group Ab: Air-blow to remove blood contamination before surface treatment.

2. Group Wb: Water-rinse to remove blood contamination before surface treatment.

3. Group Aa: Air-blow to remove blood contamination after surface treatment.

4. Group Wa: Water-rinse to remove blood contamination after surface treatment.

5. Group RT: Surface re-treatment following water-rinse to remove blood contamination after surface treatment.

6. Group NC: No blood contamination.

The surface treatment of dentin was carried out with an aqueous solution of $10 \%$ citric acid and $3 \%$ ferric chloride (10-3 solution; activator Green ${ }^{\circledR}$, Sun Medical, Shiga, Japan) for $5 \mathrm{~s}$, and the surface was rinsed with water for $20 \mathrm{~s}$ and air-dried. Blood contamination was carried out as follows: the exposed dentin surface was immediately covered with blood taken from the fingertip with lancet (Singlestick ${ }^{\circledR} \mathrm{II}$, Misawa Medical Industry, Tokyo, Japan) before or after the surface treatment, and the contaminant was left on the surface for $15 \mathrm{~s}$. After $15 \mathrm{~s}$ of exposure, the contaminated surfaces were strongly air-blown, rinsed with water for $15 \mathrm{~s}$, or additively re-treated with the 10-3 solution for $5 \mathrm{~s}$ after they were rinsed with water to remove the contaminants. The specimens that were not contaminated were used as controls.

\section{SEM observation of the dentin surface}

Thirty-six specimens were prepared for observation of the dentine surface after polishing, just after blood contamination or just before applying SB, from each group previously described. These specimens were fixed with $2.5 \%$ glutaraldehyde in $0.1 \mathrm{M}$ sodium cacodylate buffer ( $\mathrm{pH} 7.4$ ) overnight at $4^{\circ} \mathrm{C}$, then rinsed 3 times in a $0.1 \mathrm{M}$ sodium cacodylate buffer for $20 \mathrm{~min}$ per rinse and in water for $1 \mathrm{~min}$, followed by dehydration in a graded series of ethanol for 20 min. Each specimen was dried in a critical point dryer (HCP-1, Hitachi, Tokyo, Japan), sputter-coated with Pt-Pd (Ion sputter E-1030, Hitachi, Tokyo, Japan) for $120 \mathrm{~s}$, and then observed using an FE-SEM (S-4000, Hitachi, Tokyo, Japan) at a voltage of $10 \mathrm{kV}$.

\section{Dye leakage test}

Sixty specimens ( $n=10$ per group) were subjected to the dye leakage test. SB was applied on the treated surfaces of each group according to the manufacturer's instructions using a bulk-mix technique. The diameter of the dipped resin was $4 \mathrm{~mm}$. All specimens were stored in distilled water at $37^{\circ} \mathrm{C}$. After $24 \mathrm{~h}$, the specimens were transferred from the storage water into $0.5 \%$ basic fuchsine solution and kept at room temperature for $24 \mathrm{~h}$. After being rinsed with water and air-dried, they were embedded in epoxy resin (EpoFix/SpeciFix-20, Struers, Ballerup, Denmark). Then, a section was made at the center of the resin perpendicular to the bonding interface.
The length of dye penetration along the interface from periphery was measured under the light microscope (BX50, Olympus, Tokyo, Japan). The length of dye penetration was recorded for each specimen.

The data were analyzed by One-way ANOVA, Games-Howell test at a 5\% level of significance using a software package (SPSS 10.0J ${ }^{\circledR}$, SPSS, Chicago, IL, USA).

\section{SEM observation of the adhesive interface}

Two specimens per group were randomly selected after the dye leakage test. Each specimen was sectioned perpendicularly to the bonding interface to a thickness of $1 \mathrm{~mm}$. These specimens were subsequently polished with silicon carbide papers \#600, \#800, \#1000, \#1200 and \#1500 under running water, and then finished with an abrasive disk and diamond pastes (DP-Past, Struers, Ballerup, Denmark) with particle sizes of 6, 3, 1 and 0.25 $\mu \mathrm{m}$. They were cleaned ultrasonically in distilled water for $2 \mathrm{~min}$, dried at room temperature for $24 \mathrm{~h}$, Pt-Pd sputter-coated for $240 \mathrm{~s}$, and then examined using an FE-SEM at a voltage of $10 \mathrm{kV}$.

\section{Microtensile bond strength test}

Forty-eight specimens ( $n=12$ per group) were examined for microtensile bond strength (MTBS). A PMMA rod was attached to each specimen with SB. All specimens were stored in distilled water at $37^{\circ} \mathrm{C}$ for $24 \mathrm{~h}$. Each specimen, approximately $1 \mathrm{~mm}^{2}$ in cross-section, was cut perpendicularly to the bonded surface. The bonded area of each specimen was measured by means of a digital micrometer (Mitutoyo CD-15C, Mitutoyo, Kawasaki, Japan). The specimens were then attached to the testing apparatus using a cyanoacrylate adhesive (Model Repair II Blue, Dentsply-Sankin Industry, Ohtawara, Japan) and subjected to microtensile bond testing in a tabletop material tester (EZ Test, Shimadzu, Kyoto, Japan) with a cross-head speed of $1.0 \mathrm{~mm} / \mathrm{min}$. MTBS (in MPa) was derived by dividing the applied maximum force (N) resulting in fracture by the bonded area $\left(\mathrm{mm}^{2}\right)$.

The data were analyzed by One-way ANOVA, Scheffé's test at a 5\% level of significance using a software package (SPSS 10.0J ${ }^{\circledR}$, SPSS, Chicago, IL, USA).

\section{Fracture analysis}

After the MTBS test, the fractured surfaces of the dentin side were observed using SEM to evaluate morphological differences. The specimens were air-dried, mounted on an aluminum stub, Pt-Pd sputter-coated for $180 \mathrm{~s}$, and then examined using an FE-SEM at a voltage of $10 \mathrm{kV}$.

\section{RESULTS}

SEM observation of dentin surface after blood contamination

The ground dentin surface after being polished was covered with a smear layer (Fig. 2a). When blood contamination on the ground dentin surface was air-blown before surface treatment with 10-3 solution (group $\mathrm{Ab}$ ), the dentin surface was covered with 
numerous residues, round-shaped components like erythrocytes and filamentous form components resembling fibrin (Fig. 2b). When the surface was rinsed with water (group $\mathrm{Wb}$ ), blood contaminants were mostly removed but dentinal tubules were obstructed by smear plugs similar to the ground dentin surface (Fig. 2c).

In group $\mathrm{NC}$, the dentinal tubules were wide open on the ground dentin surface after the surface treatment (Fig. 2d). When the blood contamination was air-blown, and treated with 10-3 solution (group Ab), none of the contaminants remained and the dentinal tubules were slightly narrow (Fig. 2e). When the contaminated dentin surface was rinsed with water and treated with 10-3 solution (group $\mathrm{Wb}$ ), the dentinal tubules were open, but smaller than that of group NC (Fig. 2f).

When the blood contamination was carried out after surface treatment and then air-blown (group Aa), the dentin surface was covered with a thick layer of blood contaminants (Fig. 2g). After the blood was rinsed with water (group Wa), blood corpuscle elements were
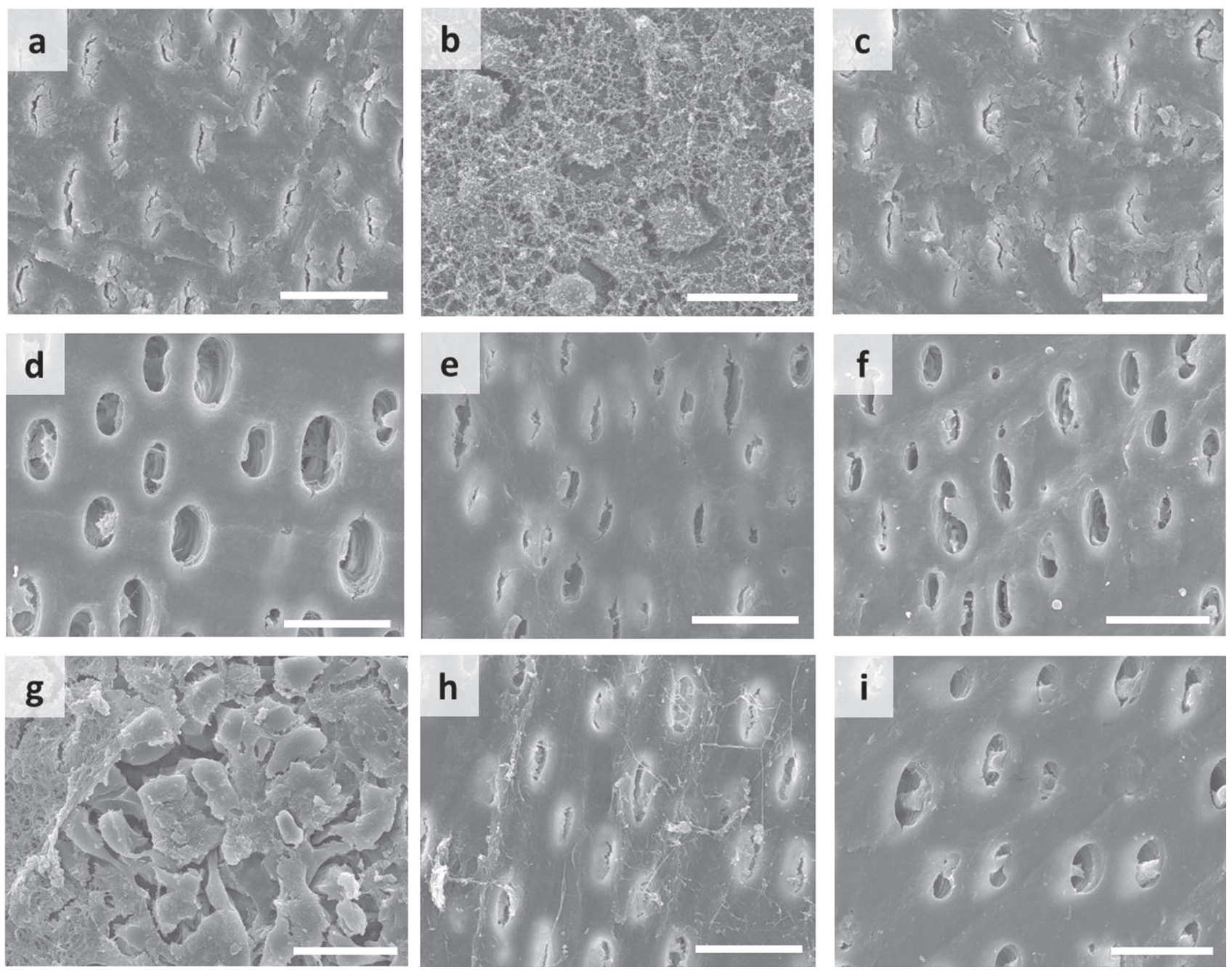

Fig. 2 SEM photographs of the dentin surfaces. (a) Ground dentin surface: The surface was covered with smear layer. (b) Blood-contaminated surface before treatment (the blood was air-blown): The surface was covered with a number of blood components resembling fibrin and hemocytes. (c) Blood-contaminated surface before treatment (the blood was rinsed with water and air-dried): The surface was covered with smear layer. (d) Group NC (surface after treatment with 10-3): The dentinal tubules were wide open. (e) Group Ab (blood was air-blown, followed by surface treatment with 10-3): The dentinal tubules were slightly narrow. (f) Group Wb (blood was rinsed with water and air-dried, followed by surface treatment with 10-3): The dentinal tubules were open, but smaller than that of control. (g) Group Aa (blood-contamination after surface treatment was air-blown): The surface was covered with a thick layer of blood contaminants. (h) Group Wa (blood-contamination after surface treatment was rinsed with water and air-dried): The dentinal tubules were closed and narrow, covered with residues resembling fibrin. (i) Group RT (blood-contaminated surface was re-treated with 10-3): Slight residues of blood contaminants were observed on the dentin surface, but the dentinal tubules were apparent. Bar: $10 \mu \mathrm{m}$. Original magnification $\times 3,000$. 


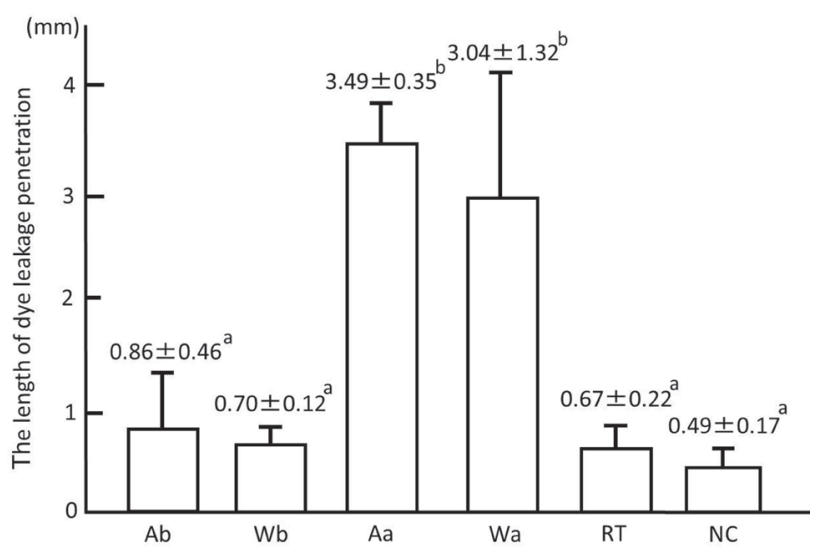

Fig. 3 The length of dye leakage penetration. The leakage values of group $\mathrm{Aa}$ and $\mathrm{Wa}$ were significantly higher compared with that of group NC. Same letter revealed no significant difference $(p<0.05)$. The results are expressed as mean \pm standard deviation (SD). Statistical differences in each group were analyzed using one-way ANOVA, Games-Howell test $(n=10$ per group).
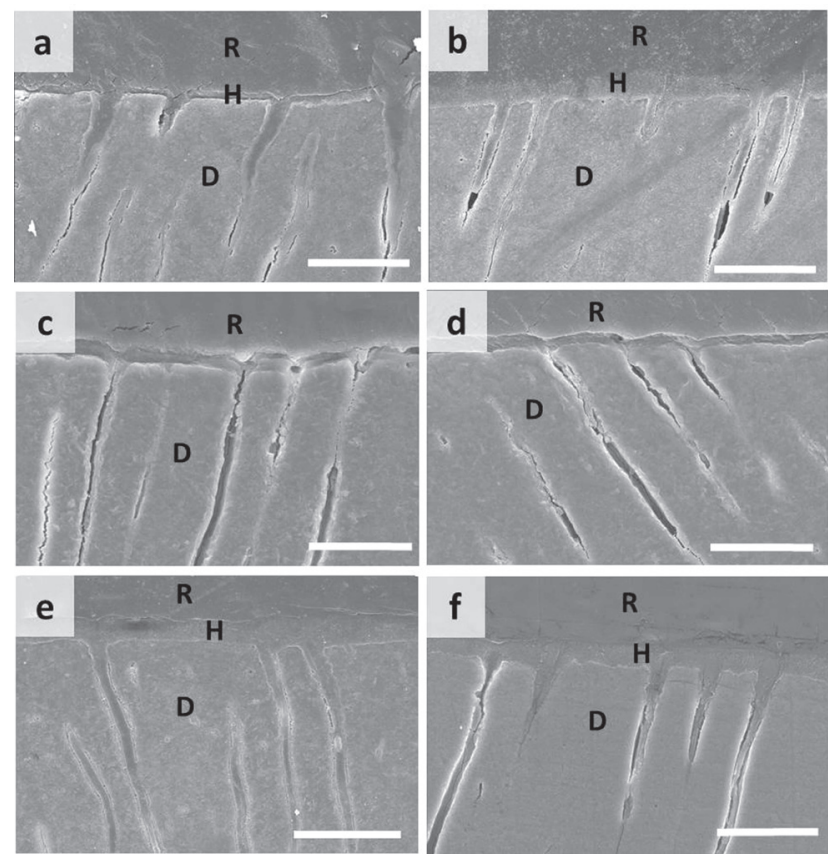

Fig. 4 SEM photographs of the adhesive interface. (a) Group Ab, (b) Group Wb, (c) Group Aa, (d) Group Wa, (e) Group RT, (f) Group NC. R: 4-META/ MMA-TBB resin, H: Hybrid layer, D: Dentin. Bar: $10 \mu \mathrm{m}$. Original magnification $\times 3,000$.

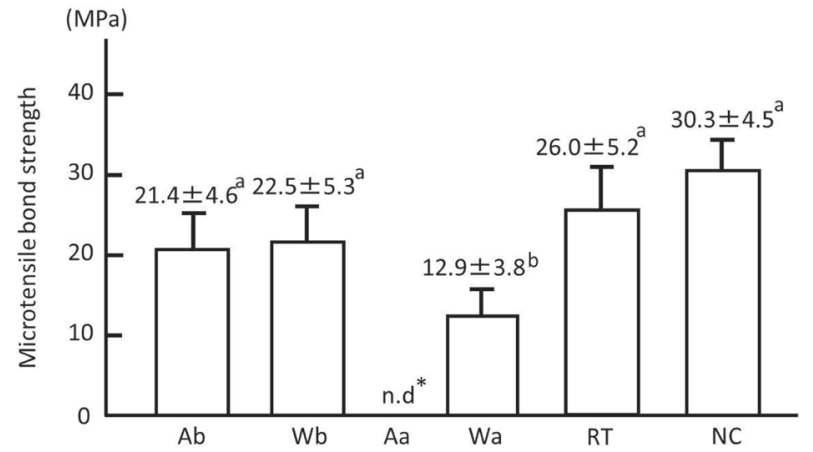

Fig. 5 Microtensile bond strength. The MTBS of group Wa was significantly lower compared with that of NC group. *: Not detected because all the specimens debonded during preparation for the MTBS test. Same letter revealed no significant difference $(p<0.05)$. The results are expressed as mean \pm standard deviation (SD). Statistical differences in each group were analyzed using one-way ANOVA, Scheffé's test ( $n=12$ per group).

removed, but filamentous residues were still observed and a large number of dentinal tubules were closed (Fig. $2 \mathrm{~h})$. However, on subsequent re-treatment of the surface (group RT), the dentinal tubules were opened and only a few, residual blood contaminants were observed (Fig. 2i).

Dye leakage assessment

The leakage values of group Aa and Wa were significantly higher than that of group NC $(p<0.05)$, although a significant difference was not observed among groups $\mathrm{Ab}, \mathrm{Wb}, \mathrm{RT}$ and NC (Fig. 3).

\section{SEM observation of the adhesive interface}

There were no spaces and hybrid layer at the adhesive interface in groups $\mathrm{Ab}, \mathrm{Wb}, \mathrm{RT}$ and NC. However, a gap was formed at the interface in groups Aa and Wa (Fig. 4).

\section{Microtensile bond strength measurement}

The MTBS of group Wa was significantly lower than that of group NC $(p<0.05)$, although a statistically significant difference was not observed among groups $\mathrm{Ab}, \mathrm{Wb}, \mathrm{RT}$ and NC (Fig. 5). The MTBS of group Aa could not be determined because all the specimens debonded during preparation for the MTBS test.

\section{Fracture analysis}

According to fractographical analysis (Fig. 6), in group $\mathrm{Ab}$ and $\mathrm{Wb}$, the incidence of cohesive failure was similar to that of mixed failure and only a few specimens showed adhesive failure. In group Aa, only adhesive failures were found. In group $\mathrm{Wa}$, the incidence of adhesive failure was almost the same as that of mixed failure and 

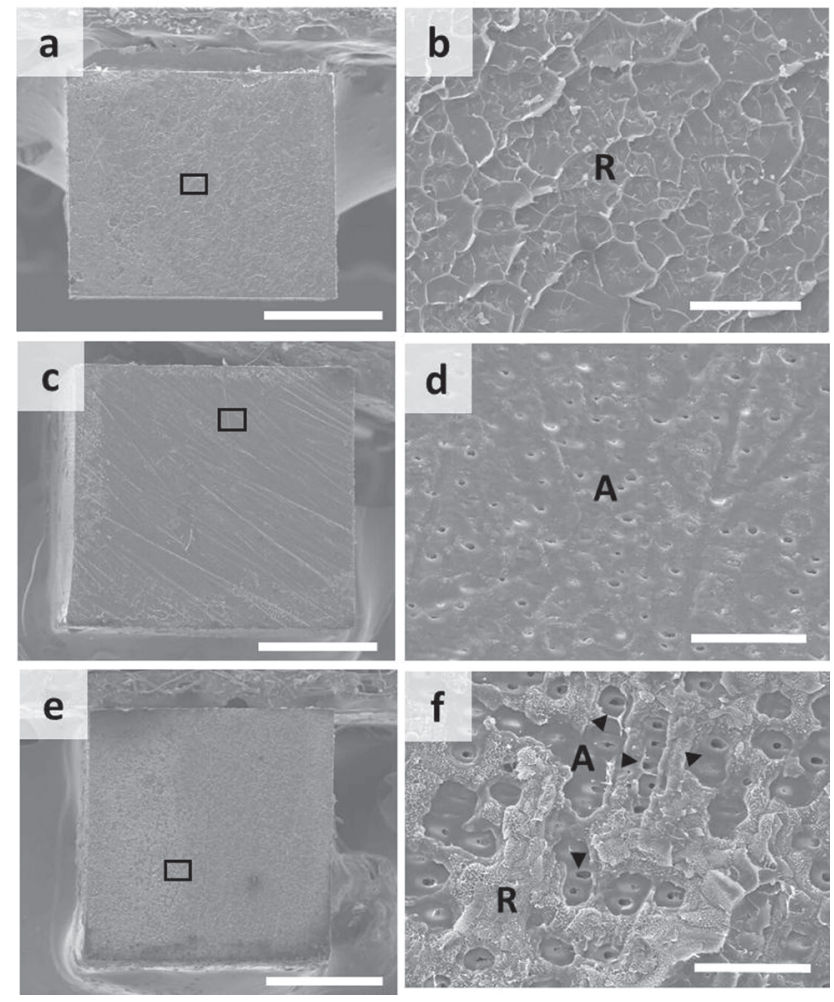

Fig. 6 SEM pictures of the fractured surfaces of dentin. (a) Cohesive failure in resin, (b) Higher magnification of squared area in a, (c) Adhesive failure at resin-dentin interface, (d) Higher magnification of squared area in c, (e) Adhesive failure and cohesive failure, (f) Higher magnification of squared area in e. R: Cohesive failure in resin, A (arrowhead): Adhesive failure. Bar: a, c and $e=500 \mu \mathrm{m} ; \mathrm{b}, \mathrm{d}$ and $\mathrm{f}=30 \mu \mathrm{m}$. Original magnification: $a, c$ and $e, \times 60 ; b, d$ and $f$, $\times 1,000$.

there were no cases of cohesive failure. Most specimens in groups RT and NC showed cohesive failure within the resin (Fig. 7).

\section{DISCUSSION}

In this study, the influence of blood contamination on adhesion of 4-META/MMA-TBB resin was evaluated, because although it has been recently applied to many surgical procedures where blood contamination often occurs, not much investigation had been carried out ${ }^{5,6}$. Fresh peripheral blood without anticoagulants was used as the contaminant in this study, whereas blood with anticoagulant was used in some experiments ${ }^{7-9,10,12)}$. This is because blood coagulation might be an important factor in determining the influence that blood contamination has on bonding ${ }^{11)}$. We evaluated the influence of blood contamination before and after surface treatment with 10-3 solution in this study, because the adhesion property may be affected by acid etching with

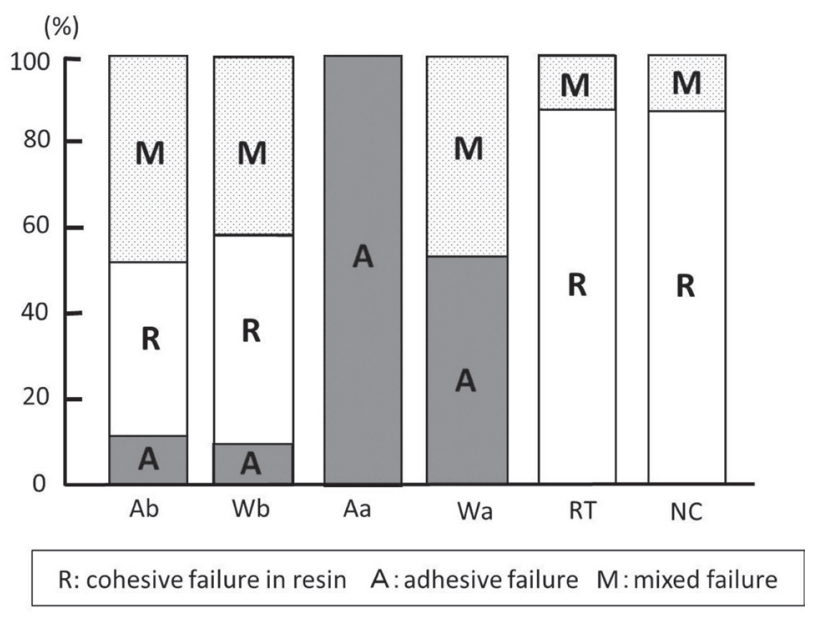

Fig. 7 Percentages of fracture modes of the fractured surface.

10-3 solution that exposes dentinal collagen fibers ${ }^{1,23,24)}$.

When blood contamination occurred before surface treatment with 10-3 (group Ab and Wb), MTBS was slightly lower compared with that of group NC (no significance was recognized) and high incidences of not only cohesive failure but also mixed failure were recognized in the fracture analysis in the present study. SEM images of group $\mathrm{Wb}$ showed that most of the blood contaminants were removed by rinsing with water and dentinal tubules were smaller than that of group NC following treatment with 10-3 solution. In group Ab after air-blowing, numerous blood contaminants were observed on the SEM images. However, after treatment with 10-3 solution, SEM images showed none of the contaminants and the dentinal tubules were open enough and slightly smaller than that of group $\mathrm{Wb}$. Furthermore, the leakage values of group $\mathrm{Ab}$ and $\mathrm{Wb}$ did not show significant differences compared with that of group NC, and no spaces and hybrid layer were observed at the adhesive interface in the present study. These results indicate that the $10-3$ solution can reach and demineralize the dentin surface even if residues remain over the dentin surface, and that blood contamination on the untreated dentin surface was almost completely eliminated, together with smear layer, by surface treatment with 10-3 solution. Therefore, blood contamination before surface treatment may have less influence on leakage and bond strength.

On the other hand, when blood contamination occurred after surface treatment followed by blown air or water-rinse (group Aa and Wa), the leakage value was significantly higher compared with that of group NC. SEM images in group Aa showed that the surface was covered with a thick layer of blood components resembling blood corpuscles and fibrin. In group Wa, MTBS was also significantly lower compared with group $\mathrm{NC}$, the incidence of adhesive failure increased compared with that of group NC and SEM images showed that most blood corpuscles were eliminated but filamentous 
residues remained and the dentinal tubules were narrow or closed even after rinsing. Shiraishi confirmed that the protein and lipid derived from blood remained on the dentin surface even after rinsing blood contaminants with water for $15 \mathrm{~s}$ using the leucomalachite green test and observation by confocal laser scanning microscopy ${ }^{9}$. In the present study, blood contaminants were rinsed with water for $15 \mathrm{~s}$ in the same way and the influence of blood contamination on dentin bonding was similar to what they had observed. These findings indicate that it is difficult to remove the blood contaminants completely after surface treatment and obtain efficient adhesion only by rinsing with water, because blood components (i.e., filamentous residues) might be strongly attached to the superficial layer of exposed collagen and the contaminant may inhibit monomer infiltration into the dentin and polymerization. Leite et al. observed that collagen network exposure by citric acid solution would lead to a more stable fibrin network and blood cell attachment to the dentin surface ${ }^{25)}$. Therefore, blood contamination after surface treatment remarkably reduces the bond strength and sealing ability.

In addition, when the surface was re-treated with 10-3 solution after rinsing the blood contaminants with water, open dentinal tubules and almost none of the filamentous or corpuscle residues were observed on SEM images. The leakage, MTBS, high incidence of cohesive failure, and the adhesive interface of group RT were similar to that of group NC. These results reveal that good adhesion was achieved after re-treatment with 10-3 solution, although some studies have reported that re-etching reduced the bond strength ${ }^{8,26)}$. These inconsistent results are attributed to several factors such as the different acid (phosphoric acid, maleic acid, citric acid) used, the total period of etching, and the bonding system applied. 10-3 solution consists of $10 \%$ citric acid ( $\mathrm{pH} 0.86$ ) and 3\% ferric chloride. Citric acid is known to have protein removal ability and anticoagulant action with its calcium chelating property and is used in clinical medicine for blood sampling, and central dialysis fluid delivery systems for hemodialysis (heat citric acid disinfection method) ${ }^{27)}$. The anticoagulant might be efficacious in removing the blood components. With an increase in the etching time, the depth of demineralised dentin is increased, resulting in reduction of the adhesive property $^{23,24,28)}$. In this study, the period of surface treatment with 10-3 solution was set to $5 \mathrm{~s}$. The total treatment time of 5-10 s was the manufacturer's recommendation. This made penetration of 4-META/ MMA-TBB resin possible through the entire demineralized dentin reaching the underlying intact dentin and initiating polymerization ${ }^{24,29)}$, and thus demineralized dentin without immersed 4-META/ MMA-TBB resin may not remain. As mentioned, re-treatment with 10-3 solution (5 s) used in this study is thought to be a reasonable method to restore the adhesive properties.

The result of this study showed that the adhesive properties were maintained by rinsing the blood with water if the blood contamination occurred before surface treatment. In contrast, when the treated surface was contaminated with blood, the surface had to be rinsed with water, followed by re-treatment with 10-3 solution.

However, blood coagulation may affect the elimination of contaminants. Further investigations on the influence of the duration of blood contamination and the durability of bonding are necessary.

\section{CONCLUSION}

If blood contamination occurred before surface treatment with 10-3 solution, washing the blood away reduced the effect of contamination on adhesion of 4-META/ MMA-TBB resin to dentin. On the other hand, blood contamination after surface treatment significantly decreased the adhesion even if the blood was washed away. It is recommended that the contaminated surface be thoroughly rinsed with water and retreated with 10-3 solution.

\section{ACKOWLEDGMENTS}

We are grateful to our colleagues Saori Tanaka Ph.D., and Ms. Mai Morikawa for their invaluable technical assistance. The authors report no conflicts of interest related to this study.

\section{REFERENCES}

1) Nakabayashi N, Kojima K, Masuhara E. The promotion of adhesion by the filtration of adhesion by the infiltration of monomers into tooth substrates. J Biomed Mater Res 1982; 16: $265-273$.

2) Nakabayashi N, Takarada K. Effect of HEMA on bonding to dentin. Dent Mater 1992; 8: 125-130.

3) Nakabayashi H, Ashizawa M, Nakamura M. Identification of a resin-dentin hybrid layer in vital human dentin created in vivo: Durable bonding to vital dentin. Quintessence Int 1992; 23: 135-141.

4) Pashley DH, Carvalho RM. Dentin permability and dentin adhesion. J Dent 1997; 25: 355-372.

5) Miles DA, Anderson RW, Pashley DH. Evaluation of the bond strength of dentin bonding agents used to seal resected root apices. J Endod 1994; 20: 538-541.

6) Vignaroli PA, Anderson RW, Pashley DH. Longitudinal evaluation of the microleakage of dentin bonding agents used to seal resected root apices. J Endod 1995; 21: 509-512.

7) Xie J, Powers JM, McGuckin RS. In vitro bond strength of two adhesives to enamel and dentin under normal and contaminated conditions. Dent Mater 1993; 9: 295-299.

8) Morikawa M, Narikawa K. The influence of blood contamination on adhesion of dentin and composite resin. Jpn J Conserv Dent 1996; 39: 168-179.

9) Shiraishi M. Analysis of blood-contaminated dentin surface and the influence of blood contamination on adhesive restorations. Jpn J Conserv Dent 1998; 41: 236-252.

10) Kaneshima T, Yatani H, Kasai T, Watanabe EK, Yamashita A. The influence of blood contamination on bond strengths between dentin and an adhesive resin cement. Oper Dent 2000; 25: 195-201.

11) Dietrich T, Kraemer MLJ, Roulet JF. Blood contamination and dentin bonding effect of anticoagulant in laboratory studies. Dent Mater 2002; 18: 159-162.

12) Shiraishi M, Tanimori H, Iwamoto K, Fujito H, Inoue M. Adhesion of composite resin to blood contaminated dentin 
-effect of contaminants on dentin pretreatment-. Jpn J Conserv Dent 2003; 46: 604-612.

13) de Carvalho Mendonça EC, Vieira SN, Kawaguchi FA, Powers J, Matos AB. Influence of blood contamination on bond strength of a self-etching system. Eur J Dent 2010; 4: 280-286.

14) Kiyomura M. Bonding strength to bovine dentin with 4-META/MMA-TBB resin - long-term stability and influence of water. J Jpn Soc Dent Mater Dev 1987; 6: 860-872.

15) Inoue $T$, Miyakoshi $S$, Shimono $M$. The in vitro and in vivo influence of 4-META/MMA-TBB resin components on dental pulp tissues. Adv Dent Res 2001; 15: 101-104.

16) Fujisawa S, Atsumi T. Cytotoxicities of a 4-META/ MMA-TBBO resin against human pulp fibroblasts. Dent Mater J 2004; 23: 106-108.

17) Sakai T, Morita S, Shinomiya K, Watanabe A, Nakabayashi N, Ishihara K. Prevention of fibrous layer formation between bone and adhesive bone cement: In vivo evaluation of bone impregnation with 4-META/MMA-TBB cement. J Biomed Mater Res 2000; 52: 24-29.

18) Nakamura M, Inoue T, Shimono M. Immunohistochemical study of dental pulp applied with 4-META/MMA-TBB adhesive resin after pulpotomy. J Biomed Mater Res 2000; 51: 241-248.

19) Ohtani K, Sugaya T, Miyaji H, Tomita M, Hasegawa $Y$, Motoki Y, Takanawa Y, Tanaka S, Kawanami M. Healing of experimental apical periodontitis after apicoectomy using different sealing methods on resected root end. Dent Mater $\mathrm{J}$ 2011; 30: 485-492.

20) Yoshimine $Y$, Ono M, Akamine A. In vitro comparison of the biocompatibility of mineral trioxide aggregate, 4-META/ MMA-TBB Resin, and intermediate restorative material as root-end-filling materials. J Endod 2007; 33: 1066-1069.

21) Masaka N. Long-term observation of fractured tooth roots preserved by adhesion. Adhes Dent 1995; 13: 156-170.

22) Sugaya T, Kawanami M, Noguchi H, Kato H, Masaka N. Periodontal healing after bonding treatment of vertical root fracture. Dent Traumatol 2001; 17: 174-179.

23) Piemjai M, Nakabayashi N. Effect of dentin conditioners on wet bonding of 4-META/MMA-TBB resin. J Adhes Dent 2001; 3: 325-331.

24) Nakabayashi N, Watanabe A, Arao T. A tensile test to facilitate identification of defects in dentine bonded specimens. J Dent 1998; 26: 379-385.

25) Leite FR, Sampaio JE, Zandim DL, Dantas AA, Leite ER, Leite AA. Influence of root-surface conditioning with acid and chelating agents on clot stabilization. Quintessence Int 2010; 41: 341-349.

26) Hiranuma K, Watanabe I, Nakabayashi N. Effect of saliva contamination on adhesion of 4-META/MMA-TBB resin to teeth penetrated with 10-3. Adhes Dent 1992; 10: 197-202.

27) Sakuma K, Uchiumi N, Sato S, Aida N, Ishimatsu T, Igoshi T, Kodama Y, Hotta H. Experience of using heat citric acid disinfection method in central dialysis fluid delivery system. J Artif Organs 2010; 13: 145-150.

28) Hashimoto M, Ohno H, Kaga M, Sano H, Tay FR, Oguchi H, Araki Y, Kubota M. Over-etching effects on micro-tensile bond strength and failure patterns for two dentin bonding systems. J Dent 2002; 30: 99-105.

29) Piemjai M, Watanabe A, Iwasaki Y, Nakabayashi N. Effect of remaining demineralised dentin on dental microleakage accessed by a dye penetration: how to inhibit microleakage? J Dent 2004; 32: 495-501. 